der Haest, Wynberg, Leusen \& Bruggink (1990). Previous crystal structure determinations of related compounds include Kok, Wynberg. Smits, Beurskens \& Parthasarathi (1987); Smits, Beurskens, Kok \& Wynberg (1987); Smits, Beurskens, Parthasarathi, Rijk, Kok \& Wynberg (1987); Kok, Wynberg, Parthasarathi, Smits \& Beurskens (1987); and Bruins Slot, Leusen, van der Haest \& Smits, 1992). Salt (1) may be called FINAM, and salt (2) FINAP." The resolving power of the phosphorinane agents in these compounds is described by van der Haest et al. (1990).

A detailed description of the crystal packing, to identify interactions which determine the differences in resolution efficiency of the resolving agent for a pair of diastereomeric salts, has been published (Leusen, Bruins Slot, Noordik, van der Haest, Wynberg \& Bruggink, 1991). A second paper on this subject will be published shortly by the same authors.

This investigation was supported (FJJL \& ADvdH) by the Netherlands Foundation of Technical Research (STW) with financial aid from

\footnotetext{
* These names are composed of the general code INA, used for all compounds in the mentioned series, followed by $M$ or $P$, indicating the two diastereomers of a pair (minus and plus rotation of plane polarized light). Eventual substituents at the aromatic moiety of the acid are indicated by prefixes, like $\mathrm{F}$.
}

the Netherlands Organization for the Advancement of Pure Research (NWO). Use of the services and facilities of the Dutch CAOS,CAMM Center, under grant Nos. SON-326-052 and STW-NCH99.1751, is gratefully acknowledged.

\section{References}

Bolhuis, F. van (1971). J. Appl. Cryst. 4, 263-264.

Bruins Slot, H. J., Lelisen, F. J. J.. van der Haest, A. D. \& Smits, J. M. M. (1992). .Acta Cryst. C48, 372-376.

Enraf-Nonius (1985). SDP PDP. Enraf-Nonius and B. A. Frenz \& Associates, Inc., Delft. The Netherlands.

Haest, A. D. VAn der, Wynberg. H., Leusen, F. J. J. \& Bruggink, A. (1990). Recl Trav. Chim. Pays-Bas, 109, 523-528. Kok, A. M. G., Wynberg, H., Parthasarathi, V., Smits, J. M. M. \& Beurskens, P. T. (1987). Acta Cryst. C43, 1336-1341.

KoK, A. M. G., WYnberg, H., Smits, J. M. M., Beurskens, P. T. \& Parthasarathi, V. (1987). Acta Cryst. C43, 1328-1331.

Leusen, F. J. J., Bruins Slot, H. J., NoOrdik, J. H., VAN DER Haest, A. D., Wynberg, H. \& Bruggink, A. (1991). Recl Trav. Chim. Pays-Bas, 110, 13-18.

Nes, G. J. H. van \& van Bolmuis, F. (1978). J. Appl. Cryst. 11, 206-207.

SheldRICX, G. M. (1976). SHELX76. Program for crystal structure determination. Univ. of Cambridge, England.

Smits, J. M. M., Beurskens, P. T., KoK, A. M. G. \& Wynberg, H. (1987). Acta Cryst. C43, 1331-1333.

Smits, J. M. M., Beurskens, P. T., Parthasarathi, V., Ruj, E. A. V., KoK, A. M. G. \& WynberG, H. (1987). Acta Cryst. C43, $1334-1336$.

SPEK, A. L. (1982). The EUCLID Package. In Computational Crystallography, edited by D. SAYRE. Oxford: Clarendon Press.

Acta Cryst. (1992). C48, 590-592

\title{
Structure of a Nucleoside Analogue 2',3'-Dideoxy-3',5-difluorouridine*
}

\author{
By D. H. Everaert, O. M. Peeters, N. M. Blaton and C. J. De Ranter $†$ \\ Laboratorium voor Analytische Chemie en Medicinale Fysicochemie, Instituut voor Farmaceutische \\ Wetenschappen, Katholieke Universiteit Leuven, Van Evenstraat 4, B-3000 Leuven, Belgium
}

\section{and A. Van Aerschot and P. HerdewiJn}

Laboratorium voor Farmaceutische Chemie, Rega Institute for Medical Research, Katholieke Universiteit Leuven, B-3000 Leuven, Belgium

(Received 5 July 1991; accepted 17 September 1991)

Abstract. 1-(2,3-Dideoxy-3-fluoro- $\beta$-D-erythro-pentofuranosyl)-5-fluorouracil. $\quad \mathrm{C}_{9} \mathrm{H}_{10} \mathrm{~F}_{2} \mathrm{~N}_{2} \mathrm{O}_{4}, \quad M_{r}=$ 248.19, monoclinic, $P 2_{1}, \quad a=5.8653(2), \quad b=$ 11.1843 (7), $c=15.9975$ (12) $\AA, \beta=93.308$ (4),$V=$

* Structural Studies of Modified Nucleosides. XV. Part XIV: De Winter. De Ranter. Blaton, Peeters, Van Aerschot \& Herdewijn (1992)

+ To whom correspondence should be addressed.

0108-270192 030590-03\$03.00
1047.7 (1) $\AA^{3}, Z=4, D_{m}=1.58, D_{x}=1.574 \mathrm{Mg} \mathrm{m}^{-3}$, Ni-filtered $\mathrm{Cu} K \alpha$ radiation, $\lambda=1.54178 \AA, \mu=$ $1.250 \mathrm{~mm}^{-1}, \quad F(000)=512, T=298 \mathrm{~K}$, final $R=$ 0.049 for 1685 unique observed reflections $[F>$ $6 \sigma(F)]$. The asymmetric unit contains two molecules $A$ and $B$. For molecule $A$, the $N$-glycosidic torsion angle $\chi$ has a value of $-167.6(4)^{\text {: }}$ in the anti range. Since the terminal $\mathrm{CH}_{2} \mathrm{OH}$ group attached to atom (C) 1992 International Union of Crystallography 
$C 4^{\prime} . A$ of the sugar moiety is found to be disordered. all data concerning sugar puckering and $\mathrm{C}^{\prime}-\mathrm{C}^{\prime}$ conformation should be considered with great caution. The sugar pucker is ${ }_{3} T^{4}$ with $P=208(1)^{\circ}$ and $\psi_{m}=29(1)^{\circ}$. Atom O5' $A$ has two different positions $\left(\mathrm{OS1}^{\prime} A\right.$ and $\left.\mathrm{O} 52^{\prime} A\right)$; for $\mathrm{O}^{\prime} 1^{\prime} A$ the $\mathrm{C} 4^{\prime}-\mathrm{C5}^{\prime}$ conformation is $+s c$ with $\gamma=62.2(9)^{\circ}$ and for O52' $A+a c$ with $\gamma=140.6(7)^{\circ}$. For molecule $B$, the $\mathrm{N}$-glycosidic torsion angle $\chi$ has a value of $-125.1(4)^{2}$ in the anti range; the sugar pucker is ${ }^{2} T_{3}$ with $P=169.3(1)^{\circ}$ and $\psi_{\mathrm{m}}=29(1)^{\circ}$ and the $\mathrm{C} 4^{\prime}-\mathrm{C}^{\prime}$ conformation is $+s c$ with $\gamma=49.1(5)^{\circ}$. All conformational parameters are in accordance with the IUPAC-IUB Joint Commission on Biochemical Nomenclature [Pure Appl. Chem: (1983), 55, 1273-1280] guidelines. Base-pair formation occurs in the crystal structure.

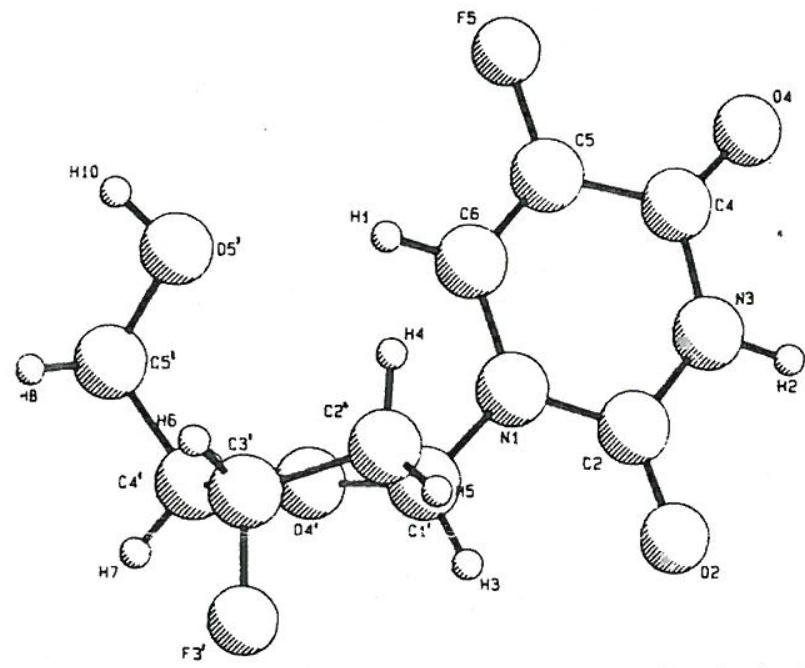

Fig. 1. PLUTO (Motherwell \& Clegg, 1978) plot of molecule $B$ with the atomic numbering scheme.

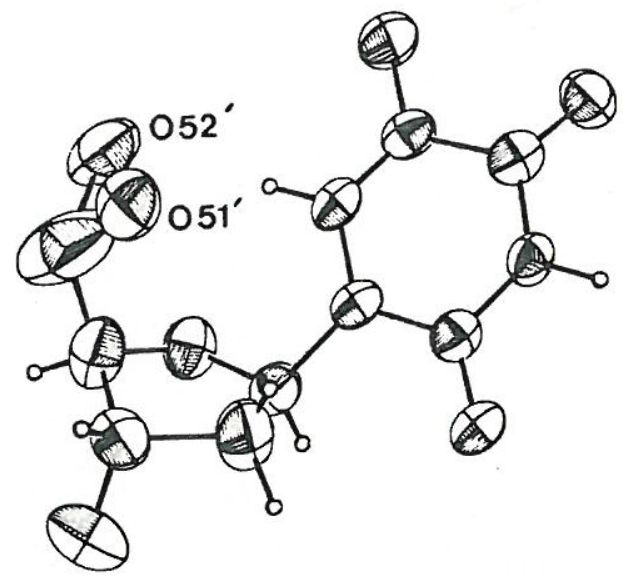

Fig. 2. ORTEP (Johnson, 1976) view of molecule $A$ with $50 \%$ probability anisotropic displacement ellipsoids for the non- $\mathrm{H}$ atoms.
Table 1. Atomic coordinates $\left(\times 10^{4}\right)$ and equivalent isotropic thermal parameters with e.s.d.'s in parentheses

$$
B_{\text {eq }}=(4 / 3) \sum i, \beta_{i j} \mathbf{a}_{i}, \mathbf{a}_{j} \text {. }
$$

\begin{tabular}{|c|c|c|c|c|}
\hline & $x$ & $y$ & $z$ & $B_{\infty}\left(A^{2}\right.$ \\
\hline $\mathrm{N} \mid A$ & $3724(6)$ & $7929^{\circ}$ & $4075(2)$ & $3.45(6)$ \\
\hline $\mathrm{C} 2 \mathrm{~A}$ & $5165(7)$ & $7772(4)$ & $3442(2)$ & $3.32(7)$ \\
\hline $02 A$ & $6863(5)$ & $7136(3)$ & $3524(2)$ & $4.28(6)$ \\
\hline $\mathrm{N} 3 \mathrm{~A}$ & $4556(6)$ & 8358 (3) & 2708 (2) & $3.49(7)$ \\
\hline $\mathrm{C} 4 \mathrm{~A}$ & $2668(7)$ & 9071 (4) & $2535(3)$ & $3.64(8)$ \\
\hline 041 & $2259(6)$ & $9510(3)$ & 1841 (2) & $4.67(7)$ \\
\hline $\mathrm{C} 5 \mathrm{~A}$ & $1290(7)$ & $9196(4)$ & $3252(3)$ & $3.75(8)$ \\
\hline F5A & $-603(5)$ & $9866(3)$ & $3142(2)$ & $5.73(7)$ \\
\hline C6 A & $1773(7)$ & $8632(4)$ & 3959 (3) & $3.69(8)$ \\
\hline $\mathrm{Cl}^{\prime} \mathrm{A}$ & $4147(8)$ & $7183(4)$ & 4846 (3) & 4.19 (9) \\
\hline$C 2.1$ & $6268(8)$ & $7567(7)$ & $5352(3)$ & $5.8(1)$ \\
\hline $\mathrm{C}^{\prime} \mathrm{A}$ & 5524 (9) & 7587 (5) & 6247 (3) & 5.0 (I) \\
\hline F3'A & $5944(9)$ & $6490(4)$ & 6615 (2) & $9.8(1)$ \\
\hline$C 4^{\prime} A$ & $3004(8)$ & $7784(6)$ & $6136(3)$ & 5.1 (1) \\
\hline 04,1 & $2295(5)$ & $7271(3)$ & 5337 (2) & $4.14(6)$ \\
\hline $\mathrm{CS}^{\prime} \mathrm{A}$ & $2250(10)$ & $9020(8)$ & $6222(5)$ & $10.3(2)$ \\
\hline $\mathrm{OSI}^{\prime} \mathrm{AT}^{+}$ & $2880(10)$ & $9652(7)$ & $5761(4)$ & $5.6(2)$ \\
\hline OS2'At & $440(10)$ & $9525(8)$ & $5692(5)$ & $7.1(2)$ \\
\hline$N \mid B$ & $-580(6)$ & 11851 (3) & $-850(2)$ & $3.32(6)$ \\
\hline$C 2 B$ & $855(7)$ & $12049(3)$ & $-1481(3)$ & $3.28(8)$ \\
\hline $\mathrm{O} 2 \mathrm{~B}$ & $2617(5)$ & 12651 (3) & $-1388(2)$ & $4.09(6)$ \\
\hline N3B & $208(6)$ & 11559 (3) & $-2246(2)$ & $3.51(7)$ \\
\hline$C 4 B$ & $-1717(7)$ & $10881(4)$ & $-2450(3)$ & $3.59(8)$ \\
\hline$O 48$ & $-2136(6)$ & $10497(3)$ & $-3147(2)$ & $4.65(7)$ \\
\hline$C 5 B$ & $-3091(7)$ & 10726 (4) & $-1738(3)$ & $3.70(8)$ \\
\hline FSB & $-5035(5)$ & $10081(3)$ & $-1877(2)$ & $5.40(6)$ \\
\hline C6B & $-2557(7)$ & $11185(4)$ & $-993(3)$ & $3.54(8)$ \\
\hline $\mathrm{Cl}^{\prime} \mathrm{B}$ & $-28(8)$ & 12326 (4) & $-12(3)$ & $3.61(8)$ \\
\hline$C 2 B$ & $-1760(10)$ & $13228(4)$ & $283(3)$ & 4.8 (1) \\
\hline$C 3^{\prime} B$ & $-1799(9)$ & 12968 (4) & $1206(3)$ & 4.7 (1) \\
\hline$F^{\prime} B$ & $-108(8)$ & $13632(3)$ & $1632(2)$ & $8.52(9)$ \\
\hline$C^{\prime} B$ & $-1198(7)$ & $11654(4)$ & 1297 (3) & $3.59(8)$ \\
\hline$O 4^{\prime} B$ & $-56(5)$ & $11349(3)$ & 544 (2) & $3.65(5)$ \\
\hline$C S^{\prime} B$ & $-3226(8)$ & $10852(5)$ & 1391 (3) & $4.26(9)$ \\
\hline$O 5^{\circ} B$ & -4955 (5) & 11113 (4) & 766 (2) & $5.27(8)$ \\
\hline
\end{tabular}

* Parameter kept fixed for origin definition.

+ Atom O5 $^{\prime} A$ is disordered.

Experimental. Colorless prismatic crystals obtained at room temperature from an ethanol-dioxane solution, dimensions $\sim 0.60 \times 0.15 \times 0.10 \mathrm{~mm}$. Density measured by flotation in $n$-heptane/CCl . Hilger \& Watts computer-controlled four-circle diffractometer, Ni-filtered $\mathrm{Cu} K \alpha$ radiation, $\omega / 2 \theta$ scan technique, $\quad 2 \theta_{\max }=130^{\circ}, \quad-7 \leq h \leq 0, \quad-13 \leq k \leq 13$, $-19 \leq l \leq 19$. Cell dimensions by least-squares refinement of the setting angles of 24 reflections with $37<2 \theta<53^{\circ}$, space group $P 2_{1}$ from systematic absences, $0 k 0$ for $k$ odd. Four standard reflections $(\overline{4} 00,020,002, \overline{2} 1 \overline{4})$ monitored after every 50 reflections showed no significant decrease in intensity per hour. 3805 reflections measured, 1822 unique reflections $\left(R_{\mathrm{int}}=0.032\right)$ of which 1687 were considered observed $[F>6 \sigma(F)]$. Two reflections $(012,121)$ badly affected by extinction were eliminated. Lorentz-polarization corrections, no absorption corrections, scattering factors from International Tables for X-ray' Crystallography (1974, Vol. IV) and for $\mathrm{H}$ atoms from Stewart, Davidson \& Simpson (1965). Structure solved with MULTAN82 (Main, Fiske, Hull, Lessinger, Germain, Declercq \& Woolfson, $1982)$. The $E$ map calculated from the solution with 
Table 2. Bond lengths (A) hond angles ( ) and selected torsion angles ( ) with e.s.d.'s in parentheses
$\mathrm{N} 1-\mathrm{C} 2$
$\mathrm{~N} 1-\mathrm{C} 6$
$\mathrm{~N} 1-\mathrm{Cl}$
$\mathrm{C} 2-\mathrm{O} 2$
$\mathrm{C} 2-\mathrm{N} 3$
$\mathrm{~N} 3-\mathrm{C} 4$
$\mathrm{C} 4-\mathrm{O} 4$
$\mathrm{C} 4-\mathrm{CS}$
$\mathrm{C} 5-\mathrm{F} 5$
$\mathrm{C} 5-\mathrm{C} 6$
$\mathrm{C} 1-\mathrm{C} 2$
$\mathrm{C} 1-\mathrm{O}$
$\mathrm{C} 2-\mathrm{C} 3$
$\mathrm{C} 3-\mathrm{F} 3$
$\mathrm{C} 3-\mathrm{C} 4$
$\mathrm{C} 4-\mathrm{O}$
$\mathrm{C} 4-\mathrm{C} 5$
$\mathrm{C} 5-\mathrm{O} 5$

$\mathrm{C} 2-\mathrm{N} 1-\mathrm{C} 6$

$\mathrm{C} 2-\mathrm{N} 1-\mathrm{Cl}^{2}$

$\mathrm{C} 6-\mathrm{N} 1-\mathrm{Cl}_{1}$
$\mathrm{~N} 1-\mathrm{C}_{2}-\mathrm{O} 2$

$\mathrm{N} 1-\mathrm{C}_{2}-\mathrm{N} 3$

$\mathrm{O} 2-\mathrm{C} 2-\mathrm{N} 3$

$\mathrm{C} 2-\mathrm{N} 3-\mathrm{C} 4$

$\mathrm{N} 3-\mathrm{C} 4-\mathrm{O} 4$

$\mathrm{O} 4-\mathrm{C} 4-\mathrm{CS}$

$\mathrm{C} 4-\mathrm{CS}-\mathrm{F} 5$

$\mathrm{C} 4-\mathrm{CS}-\mathrm{C} 6$

$\mathrm{F} 5-\mathrm{C} 5-\mathrm{C} 6$

$\mathrm{N} 1-\mathrm{C} 6-\mathrm{C} 5$

$\mathrm{N} 1-\mathrm{C} 1-\mathrm{C} 2$

$\mathrm{N} 1-\mathrm{Cl}-\mathrm{O}$

$\mathrm{C} 2^{\prime}-\mathrm{Cl}^{\prime}-\mathrm{O}^{\prime}$

$\mathrm{Cl}-\mathrm{C} 2^{\prime}-\mathrm{C}^{\prime}$

$\mathrm{C2}^{\prime}-\mathrm{C}^{\prime}-\mathrm{C4}^{\prime}$

$\mathrm{F}^{\prime}-\mathrm{C}^{\prime}-\mathrm{C}^{\prime}$

$\mathrm{C}^{\circ}-\mathrm{C} 4^{\circ}-\mathrm{O}^{\prime}$

$\mathrm{C}^{\circ}-\mathrm{C}^{2}-\mathrm{C}^{\circ}$

O4 $-\mathrm{CA}^{\prime}-\mathrm{C}^{\circ}$

$1,-04-C^{\prime}$

$4^{\prime}-\mathrm{Cs}^{\prime}-\mathrm{OS}^{\prime}$

$\mathrm{C} 2-\mathrm{N} 1-\mathrm{Cl}^{\prime}-\mathrm{O}^{\prime}$

$\mathrm{O}^{\prime}-\mathrm{Cl}^{\prime}-\mathrm{C}^{\prime}-\mathrm{C}^{2}$

$\mathrm{C} 1-\mathrm{C}^{\prime}-\mathrm{C}^{\prime}-\mathrm{C}^{\prime}$

$\mathrm{C}^{\prime}-\mathrm{C}^{\prime}-\mathrm{C}^{\prime}-\mathrm{O}^{\prime}$

$\mathrm{C}^{\prime}-\mathrm{C}^{\prime}-\mathrm{O}^{\prime}-\mathrm{C}$

(OS2')
$\mathrm{C}^{\prime}-\mathrm{C}^{\prime}-\mathrm{F}^{\prime}$

$\mathrm{C}{ }^{\prime}-\mathrm{Cl}^{\prime}-\mathrm{O}^{\prime}-\mathrm{C}^{\prime}$

$\mathrm{C}^{3}-\mathrm{C}^{2}-\mathrm{Cs}^{-}-\mathrm{O}$

$=0.049, \quad w R=0.066 . \quad S=2.703, \quad w=\left[\sigma\left(F_{o}\right)^{2}+\right.$ $\left.0.0004\left(F_{o}\right)^{2}\right]^{2}$ '. 317 refined parameters, maximum shift e.s.d. $=0.03$, min. and max. electron density -0.316 and $0.214 \mathrm{e} \AA^{-3}$. Number of reflections per refined variable was $1685 / 317=5.3$. All calculations were performed on a PDP $11 / 73$ microcomputer using $S D P$ (B. A. Frenz \& Associates, Inc., 1982) and PARST (Nardelli, 1983).

A PLUTO (Motherwell \& Clegg, 1978) plot of molecule $B$ with the atomic numbering scheme is shown in Fig. 1. An ORTEP (Johnson, 1976) view of molecule $A$ with $50 \%$ probability anisotropic displacement ellipsoids for the non- $\mathrm{H}$ atoms is shown in Fig. 2. Final fractional atomic coordinates are given in Table 1. ${ }^{*}$ Bond lengths, bond angles and selected torsion angles are given in Table 2. Except for the disordered terminal $\mathrm{CH}_{2} \mathrm{OH}$ group of molecule $A$, all values are regarded as normal.

Related literature. The method of preparation has been described by Van Aerschot, Herdewijn, Balzarini, Pauwels \& De Clercq (1989).

The authors wish to thank J. P. Van Cuyck for his help in preparing the figures.

- Lists of structure factors, anisotropic thermal parameters, bond lengths and angles involving $\mathrm{H}$ atoms, least-squares planes and $\mathrm{H}$-atom parameters have been deposited with the British Library Document Supply Centre as Supplementary Publication No. SUP 54662 (18 pp.). Copies may be obtained through The Technical Editor, International Union of Crystallography, 5 Abbey Square, Chester $\mathrm{CH}$ ] 2HU, England. [CIF reference: GE0282]

\section{References}

B. A. Frenz \& Associates, Inc. (1982). SDP Structure Determination Package. College Station, Texas, USA

De Winter, H. L., De Ranter, C. J., Blaton, N. M., Peeters, O. M., Van Aerschot, A. \& Herdewin, P. (1992). Acta Cryst. B48, 95-103.

IUPAC-IUB JOINT COMMISSION ON BIOCHEMICAL NomenClatURE (1983). Pure Appl. Chem. 55. 1273-1280.

the best figure of merit revealed 18 of the 34 non- $\mathrm{H}$ atoms in the asymmetric unit. All remaining atoms (except $\mathrm{O}^{\prime} A$ ) were obtained from a subsequent Fourier synthesis. $\mathrm{O}^{\prime} A$ was found to be disordered $\left(\mathrm{O}^{\prime} 1^{\prime} A\right.$ and $\mathrm{O}^{\prime} 2^{\prime} A, 50 \%$ each). A difference synthesis revealed the position of atom $\mathrm{H} 10 B$ (attached to atom $\left.\mathrm{O}^{\prime} B\right)$. All other $\mathrm{H}$ atoms except those bonded to atoms $\mathrm{C5}^{\prime} A$ and $\mathrm{O5}^{\prime} A$ were included at calculated positions $(\mathrm{C}-\mathrm{H}$ and $\mathrm{N}-\mathrm{H}$ distances $0.95 \AA$ ). $\mathrm{H}$ atoms were given fixed isotropic temperature factors 1.3 times that of their parent atom. All other atoms were refined anisotropically on $F$ by full-matrix least squares. Refinement converged at $R$
JoHNson, C. K (1976). ORTEPII. Report ORNL-5138. Oak Ridge National Laboratory. Tennessee, USA.

Main, P., Fiske, S. J., Hull. S. E., Lessinger, L., Germain, G. DeClerCQ, J.-P. \& WOOLFSON, M. M. (1982). MLLLTAN82. A System of Computer Programs for the Automatic Solution of Crystal Structures from X-rav Diffraction Data. Univs. of York, England, and Louvain, Belgium.

Motherwell, W. D. S. \& ClegG. W. (1978). PLLTO. Program for plotting molecular and crystal structures. Univ. of Cambridge. England.

NARDElli, M. (1983). J. Comput. Chem. 7. 95-98.

Stewart, R. F. Davidion, E. R. \& Simpson, W. T. (1965). J Chem. Phys. 42, 3175-3187.

Van Aerschot, A. Herdewin, P., Balzarini, J., Palwels, R. \& De ClercQ, E. (1989). J. Med. Chem. 32, 1743-1749. 